\title{
Many-body effects in graphene beyond the Dirac model with Coulomb interaction
}

\author{
N. Yu. Astrakhantsev, ${ }^{1,2, *}$ V. V. Braguta, ${ }^{3,4,5}$ and M. I. Katsnelson ${ }^{6,7}$ \\ ${ }^{1}$ Institute for Theoretical and Experimental Physics, Moscow 117218, Russia \\ ${ }^{2}$ Moscow Institute of Physics and Technology, Dolgoprudny 141700, Russia \\ ${ }^{3}$ Institute for High Energy Physics, NRC “Kurchatov Institute," Protvino 142281, Russia \\ ${ }^{4}$ School of Biomedicine, Far Eastern Federal University, Vladivostok 690950, Russia \\ ${ }^{5}$ National Research Nuclear University MEPhI (Moscow Engineering Physics Institute), Kashirskoe Highway, 31, Moscow 115409, Russia \\ ${ }^{6}$ Institute for Molecules and Materials, Radboud University, Heyendaalseweg 135, NL-6525AJ Nijmegen, The Netherlands \\ ${ }^{7}$ Theoretical Physics and Applied Mathematics Department, Ural Federal University, Mira Street 19, Ekaterinburg 620002, Russia
}

(Received 4 June 2015; revised manuscript received 19 October 2015; published 7 December 2015)

\begin{abstract}
This paper is devoted to the development of perturbation theory for studying the properties of graphene sheets of finite size, at nonzero temperature and chemical potential. The perturbation theory is based on the tight-binding Hamiltonian and arbitrary interaction potential between electrons, which is considered as a perturbation. One-loop corrections to the electron propagator and to the interaction potential at nonzero temperature and chemical potential are calculated. One-loop formulas for the energy spectrum of electrons in graphene, for the renormalized Fermi velocity and also for the dielectric permittivity, are derived.
\end{abstract}

DOI: 10.1103/PhysRevB.92.245105

PACS number(s): 73.22.Pr, 05.10.Ln, 11.15.Ha

\section{INTRODUCTION}

Graphene is a two-dimensional crystal composed of carbon atoms which are packed in a honeycomb (hexagonal) lattice $[1,2]$. It attracts considerable interest because of its unique electronic properties; most of them are related to the existence of two conical points in the electron energy spectrum (Fermi points) and the "massless Dirac fermion" character of its charge carriers with energy and momentum close to the Fermi points [3-7]. It results in numerous quantum relativistic phenomena such as Klein tunneling, minimal conductivity through evanescent waves, relativistic collapse at a supercritical charge, etc., establishing an interesting and fruitful relation between fundamental physics and materials science [8-13]. The effective "velocity of light" (Fermi velocity) for the Dirac fermions in graphene is relatively small, $v_{F} \sim c / 300$, and the interaction between the quasiparticles in graphene can be approximated by the instantaneous Coulomb potential with the effective coupling constant ${ }^{1} \alpha_{\text {eff }} \sim \alpha\left(c / v_{F}\right) \sim 300 / 137 \sim 2$. This interaction is therefore quite strong, which results in a rich variety of phenomena [12]. Within the Dirac model, before the experimental discovery of graphene, it was shown that the long-range character of the Coulomb interaction results in a renormalization of the Fermi velocity which is divergent at zero temperature and zero doping, leading to a non-Fermi-liquid behavior [14]; this prediction has been recently confirmed experimentally $[15,16]$.

At the same time, the Dirac model gives us a manybody renormalization of electronic properties only for small coupling constants and only with a logarithmic accuracy. Logarithmic corrections to the Fermi velocity were discussed also in a context of static and optical conductivity of undoped graphene (see, e.g., Refs. [17-20]). The higher-order terms were considered in Refs. [21,22], but still within the Dirac model. To calculate quantitatively correctly these properties one needs to work with a lattice model and with a realistic

\footnotetext{
*nikita.astrakhantsev@itep.ru

${ }^{1}$ In this paper, we work in units $\hbar=1$.
}

potential of the electron-electron interaction $V_{x y}$, taking into account its screening by $\sigma$ electrons. The corresponding first-principles results [23] can be parametrized by a phenomenological potential. This modification of the interaction potential at small scales in comparison with a bare Coulomb potential was proved to significantly affect some graphene properties (for instance, the phase diagram of graphene [24]).

The authors of Refs. [24-26] carried out a Monte Carlo study of graphene properties based on a tight-binding Hamiltonian without expansion near the Fermi points. Within this approach one can introduce an arbitrary phenomenological potential $V_{x y}$. Using a tight-binding Hamiltonian on a hexagonal lattice, we are going to build the perturbation theory in $V_{x y}$ at nonzero temperature and nonzero chemical potential. We believe that the theory built in this way is important and interesting for the following reasons.

The theory based on the tight-binding Hamiltonian has more common features with real graphene physics than the effective theory based on the expansion in the vicinity of Dirac points. For instance, the tight-binding Hamiltonian "remembers" graphene properties such as the geometry of a hexagonal lattice or the natural energy scale such as the $\pi$ bandwidth, which are absent in the effective Dirac theory. Moreover, one can include a phenomenological potential in this theory, which is closer to the real graphene physics than the bare Coulomb potential.

In addition, the theory based on the tight-binding Hamiltonian and the phenomenological interaction $V_{x y}$ can be easily improved. For instance, one can study the effects appearing due to the inclusion of next-to-nearest-neighbor hopping. Note that such a study cannot be carried out within a Monte Carlo simulation because of the well-known sign problem. Finally, if the electron properties of other nanomaterials are formulated in terms of the tight-binding Hamiltonian and the phenomenological potential, it is not difficult to apply the results of this paper to study these materials (see, for example, Ref. [27]).

Lattice simulations of graphene were proved to be a very efficient and quickly developing approach for studying the properties of graphene [24-26,28-30]. An important feature of all these simulations is that they are conducted at a finite 
lattice, at a finite temperature, and with finite discretization errors. In order to check the lattice results and estimate the discretization uncertainty in the weak coupling region, it is very useful to develop a perturbation theory which accounts for all these effects.

Strictly speaking, the theory with an arbitrary phenomenological potential is not renormalizable since it contains fourfermion terms. However, a lattice formulation provides the ultraviolet (spacing between carbon atoms) and the infrared (the finite size of the lattice) regulators. For this reason the theory on the hexagonal lattice is well defined.

Finally, we should mention that the interaction in graphene is strong, so the application of the perturbation theory is questionable. However, we believe that even at the one-loop level one can study some important physical effects. One can also expect that the perturbation theory built in this paper can well describe graphene many-body effects similarly to the random phase approximation (RPA) based on the effective theory of graphene [22].

We would like to note that there are papers where a tight-binding model was used to study graphene properties. In particular, a tight-binding model for interacting electrons on a hexagonal lattice with retarded Coulomb interactions, zero temperature, and zero chemical potential was introduced and studied in Refs. [31,32], where renormalizability at all orders and the emergence of critical exponents were proved. The other examples are Refs. [33,34], which aimed at a study of the optical conductivity using a tight-binding model.

This paper is organized as follows. In the next section, we build the perturbation theory which is based on the tight-binding Hamiltonian and an arbitrary interaction potential between electrons. In Sec. III we calculate one-loop corrections to the electron propagator. Section IV is devoted to the calculation of one-loop corrections to the interaction potential. Finally, in the last section, we discuss and summarize the results of this paper.

\section{PARTITION FUNCTION AND FEYNMAN RULES}

\section{A. Geometry}

We consider a hexagonal lattice with a torus topology. An example of such a lattice, which consists of $L_{x} \times L_{y}=6 \times 6$ hexagons, is shown in Fig. 1.

The hexagonal lattice is composed of two triangular sublattices $A$ and $B$. The sites belonging to sublattices $A$ and $B$ are shown as rectangles and circles, respectively. The Cartesian coordinates $(x, y)$ of any lattice site can be parametrized by three numbers $\left(s, \xi_{1}, \xi_{2}\right)$, where $s=A / B$ is the sublattice index and $\xi_{1}=0 \ldots L_{x}-1, \xi_{2}=0 \ldots L_{y}-1$, so

$$
\begin{gathered}
x=\sqrt{3} a \xi_{1}+\frac{\sqrt{3}}{2} a \xi_{2}+\frac{\sqrt{3}}{2} a \delta_{s, B}, \\
y=\frac{3}{2} a \xi_{2}-\frac{1}{2} a \delta_{s, B} .
\end{gathered}
$$

The torus topology implies the following identification of $\vec{\xi}$ coordinates:

$$
\left(\xi_{1}+L_{x}, \xi_{2}\right) \rightarrow\left(\xi_{1}, \xi_{2}\right), \quad\left(\xi_{1}, \xi_{2}+L_{y}\right) \rightarrow\left(\xi_{1}+L_{y} / 2, \xi_{2}\right)
$$

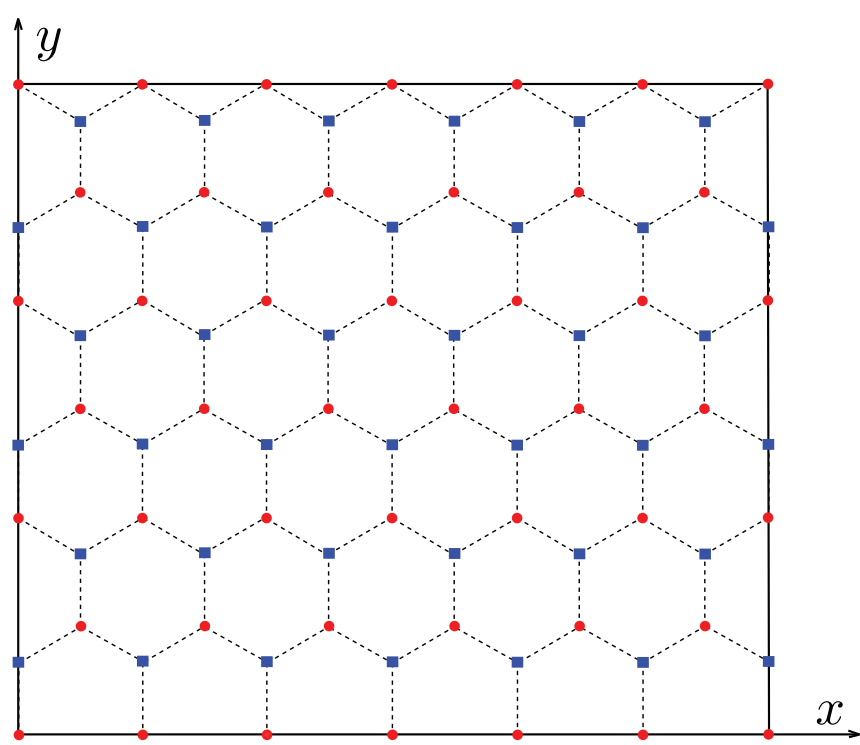

FIG. 1. (Color online) $L_{x} \times L_{y}=6 \times 6$ graphene sheet. The sites belonging to the sublattices $A$ and $B$ are shown as rectangles and circles, respectively.

Every site of the $A$ sublattice is connected to three sites of the sublattice $B$. The vectors $\vec{\rho}_{b}$ in the $\xi$ coordinates connect $(\alpha, \vec{\xi})$ to its neighbors $\left(\beta, \vec{\xi}+\vec{\rho}_{b}\right)$ :

$$
\vec{\rho}_{1}=(0,0), \quad \vec{\rho}_{2}=(-1,1), \quad \vec{\rho}_{3}=(-1,0) .
$$

In the $x$ coordinates these $\vec{\rho}_{b}$ vectors read

$$
\vec{\rho}_{1}=(0,0), \quad \vec{\rho}_{2}=\left(-\frac{\sqrt{3}}{2} a, \frac{3}{2} a\right), \quad \vec{\rho}_{3}=(-\sqrt{3} a, 0) .
$$

\section{B. Tight-binding Hamiltonian with interactions}

The electronic properties of graphene can be described by the tight-binding Hamiltonian

$$
\hat{H}_{\mathrm{tb}}=-t \sum_{\sigma=\uparrow, \downarrow} \sum_{\langle x y\rangle}\left(\hat{c}_{\sigma, x}^{\dagger} \hat{c}_{\sigma, y}+\hat{c}_{\sigma, y}^{\dagger} \hat{c}_{\sigma, x}\right),
$$

where the summation is kept over the neighboring graphene lattice sites $x$ and $y$, and $t \approx 2.7 \mathrm{eV}$ is the hopping parameter. Operators $\hat{c}_{\sigma, x}^{\dagger}, \hat{c}_{\sigma, x}$ create and annihilate electrons with spin $\sigma=\uparrow, \downarrow$ at the lattice site $x$. Note that one can also include next-to-nearest neighbors hopping to the Hamiltonian (6), but for simplicity we restrict our consideration to the nearest neighbors.

We choose the vacuum state that satisfies the following conditions,

$$
\hat{c}_{\uparrow, x}|0\rangle=0, \quad \hat{c}_{\downarrow, x}^{\dagger}|0\rangle=0,
$$

so there is an electron with spin $\sigma=\downarrow$ at every lattice site and no electrons with spin $\sigma=\uparrow$. As our main goal is to calculate the partition function $\mathcal{Z}$, which contains a summation over all states, the specific choice of $|0\rangle$ will not affect any physical results.

It is convenient to rewrite the Hamiltonian in terms of creation and annihilation operators for "particles" and "holes," 
which read

$$
\hat{a}_{x}=\hat{c}_{+, x}, \quad \hat{b}_{x}= \pm \hat{c}_{-, x}^{\dagger} .
$$

The plus sign is taken for $x \in A$ and the minus sign for $x \in B$, where $A$ and $B$ are the triangular sublattice labels. After a redefinition of the operators, the ground state satisfies $\hat{a}_{x}|0\rangle=$ $\hat{b}_{x}|0\rangle=0$. Thereby, we interpret the absence of a valence electron as a positively charged "hole" and an additional electron as a negatively charged "particle." In terms of these operators, the tight-binding Hamiltonian is

$$
\hat{H}=-t \sum_{\langle x y\rangle}\left(\hat{a}_{x}^{\dagger} \hat{a}_{y}+\hat{b}_{y}^{\dagger} \hat{b}_{x}+\text { H.c. }\right) \text {. }
$$

The charge operator now reads

$$
\hat{q}_{x}=\hat{a}_{x}^{\dagger} \hat{a}_{x}-\hat{b}_{x}^{\dagger} \hat{b}_{x} .
$$

It easy to check that $\hat{q}_{x}|0\rangle=0$, which means that this vacuum is electrically neutral.

In some applications [24,25], a mass term is introduced,

$$
\hat{H}_{m}=\sum_{x}\left( \pm m c^{2}\right) \hat{a}_{x}^{\dagger} \hat{a}_{x}+\sum_{x}\left( \pm m c^{2}\right) \hat{b}_{x}^{\dagger} \hat{b}_{x},
$$

where the plus sign is taken for sublattice $A$ and the minus sign for sublattice $B$. This term explicitly breaks the symmetry between two sublattices.

In order to study the action of the chemical potential on graphene properties, we introduce the term

$$
\hat{H}_{\mu}=\mu \sum_{x}\left(\hat{a}_{x}^{\dagger} \hat{a}_{x}-\hat{b}_{x}^{\dagger} \hat{b}_{x}\right)
$$

It is known that the interaction between electrons plays an important role. This part of the Hamiltonian has the form

$$
\hat{H}_{\text {int }}=\frac{1}{2} \sum_{x, y} V_{x y} \hat{q}_{x} \hat{q}_{y} .
$$

The Coulomb potential $V$ is often used to describe the interaction. However, it was shown in Ref. [23] that the real potential $V_{x y}$ dramatically deviates from the Coulomb law at small distances, which affects physical properties [24].

The aim of this paper is to study the properties of the electronic system, described by the Hamiltonian

$$
\begin{aligned}
\hat{H}= & -t \sum_{\langle x y\rangle}\left(\hat{a}_{x}^{\dagger} \hat{a}_{y}+\hat{b}_{y}^{\dagger} \hat{b}_{x}+\text { H.c. }\right)+\sum_{x}\left( \pm m c^{2}\right) \hat{a}_{x}^{\dagger} \hat{a}_{x} \\
& +\sum_{x}\left( \pm m c^{2}\right) \hat{b}_{x}^{\dagger} \hat{b}_{x}+\mu \sum_{x}\left(\hat{a}_{x}^{\dagger} \hat{a}_{x}-\hat{b}_{x}^{\dagger} \hat{b}_{x}\right) \\
& +\frac{1}{2} \sum_{x, y} V_{x y} \hat{q}_{x} \hat{q}_{y},
\end{aligned}
$$

where $V_{x y}$ is an arbitrary phenomenological interaction, treated as the perturbation.

\section{Electronic spectrum of graphene without interaction}

If the interaction is neglected, ${ }^{2}$ one can easily find the Hamiltonian spectrum and its eigenfunctions, which can be

\footnotetext{
${ }^{2}$ In this section we consider graphene with zero chemical potential.
}

written as

$$
\psi_{\vec{k}}^{\zeta}(\vec{x})=\left(\begin{array}{c}
c_{A}^{\zeta}(\vec{k}) \\
c_{B}^{\zeta}(\vec{k})
\end{array}\right) e^{i \vec{k} \vec{x}},
$$

where $\zeta= \pm 1$ is an additional label representing particles $(\zeta=+1)$ and antiparticles $(\zeta=-1)$. The first vector component corresponds to the $A$ sublattice, and similarly the second one corresponds to the $B$ sublattice. The Brillouin zone momentums $\vec{k}$ are

$$
k_{x}=\frac{2 \pi m_{x}}{\sqrt{3} a L_{x}}, \quad k_{y}=\frac{2 \pi m_{y}}{3 a\left(L_{y} / 2\right)} .
$$

Indices $m_{x}=0, \ldots, L_{x}-1, m_{y}=0, \ldots, L_{y}-1$ give the full set of eigenfunctions for the torus topology of the graphene sheet.

The energy spectrum of the Hamiltonian is then

$$
E_{\zeta}(\vec{k})=\zeta \sqrt{m^{2} c^{4}+|\varphi(\vec{k})|^{2}}, \quad \text { where } \quad \varphi(\vec{k})=t \sum_{b=1}^{3} e^{i \vec{k} \vec{\rho}_{b}} .
$$

The wave function components are

$$
\begin{aligned}
c_{A}^{\zeta}(\vec{k}) & =\sqrt{\frac{E(\vec{k})+\zeta m c^{2}}{2 E(\vec{k}) L_{x} L_{y}}}, \\
c_{B}^{\zeta}(\vec{k}) & =-\zeta e^{-i \arg \varphi(\vec{k})} \sqrt{\frac{E(\vec{k})-\zeta m c^{2}}{2 E(\vec{k}) L_{x} L_{y}}} .
\end{aligned}
$$

There exist two special momenta $\vec{k}_{F}$, such that $\varphi\left(\vec{k}_{F}\right)=$ 0 and $E\left(\vec{k}_{F}\right)= \pm m c^{2}$, called the Dirac points. There are two different Dirac positioned at $\left(m_{x}, m_{y}\right)=\left(2 L_{x} / 3,0\right)$ and $\left(L_{x} / 3, L_{y} / 2\right)$.

For the massless fermions $(m=0)$, in the vicinity of the Dirac points the energy spectrum of the fermion quasiparticles is

$$
\begin{aligned}
E(\vec{k}) & =v_{F}\left|\vec{k}-\vec{k}_{F}\right|, \\
v_{F}^{0} & =\frac{3}{2} t a \approx \frac{c}{343}=0.87 \times 10^{6} \frac{\mathrm{m}}{\mathrm{s}} .
\end{aligned}
$$

The linear spectrum of fermion excitations plays the central role in the effective theory of graphene.

\section{Partition function}

Let us consider a graphene sheet $L_{x} \times L_{y}$ at temperature $T$. To write down the partition function for this graphene sample, one should handle the 4-fermion interaction operator which is contained in the Hamiltonian (14). So, before going to the partition function, the interaction term of the Hamiltonian should be decomposed using the Hubbard-Stratonovich transformation

$$
\begin{aligned}
& \exp \left(-\frac{1}{2} \sum_{x, y} \hat{q}_{x} V_{x y} \hat{q}_{y}\right) \\
& =\int \mathcal{D} \varphi \exp \left(-\frac{1}{2} \sum_{x, y} \varphi_{x} V_{x y}^{-1} \varphi_{y}-i \sum_{x} \varphi_{x} \hat{q}_{x}\right) .
\end{aligned}
$$

In the last expression we introduced the Hubbard field $\varphi$, which carries the interaction. Note also that we have omitted 


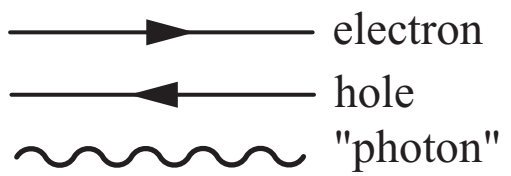

FIG. 2. Graphical representation of the propagators of different particles.

the determinant of the $V$, which is not important for our calculations.

Now, it causes no difficulties to write down the partition function in terms of the path integral. To this end, we divide the Euclidean time $\tau_{E} \in[0, \beta], \beta=1 / T$ into $N_{\tau}$ parts with size $\delta \tau=\beta / N_{\tau}$.

We introduce the electron fields $(\bar{\eta}, \eta)$, the hole fields $(\bar{\psi}, \psi)$, and the Hubbard field $\varphi$ (which is called a "photon" below) at each site of the lattice $L_{x} \times L_{y} \times \beta$. The boundary conditions in the Euclidean time direction are periodic for "photon" $\varphi$ and antiperiodic for the fermion fields $(\bar{\eta}, \eta),(\bar{\psi}, \psi)$. The boundary conditions for the spatial directions are periodic for all the fields.

Finally, the partition function for the model can be written as $[24,25]$

$$
\begin{aligned}
\mathcal{Z}= & \int \mathcal{D} \varphi \mathcal{D} \bar{\eta} \mathcal{D} \eta \mathcal{D} \bar{\psi} \mathcal{D} \psi \\
& \times e^{-\mathcal{S}_{e m}(\varphi)-\sum_{\sigma, x, y} \bar{\eta}(x) \mathcal{M}_{x, y}(\varphi) \eta(y)-\sum_{\sigma, x, y} \bar{\psi}(x) \overline{\mathcal{M}}_{x, y}(\varphi) \psi(y)}
\end{aligned}
$$

where the matrix $\mathcal{M}_{l_{1}, l_{2}, \sigma}(x, y)$ reads (here, $l_{1}=A, B$ and $l_{2}=A, B$ are sublattice indices)

$$
\begin{aligned}
& \mathcal{M}_{l_{1}, l_{2}}(x, y)=\left(\delta_{\vec{x}, \vec{y}} \delta_{l_{1}, l_{2}} \delta_{x^{0}, y^{0}}-e^{i \delta \tau \varphi_{l_{1}}(x)} e^{\delta \tau \mu} \delta_{\vec{x}, \vec{y}} \delta_{l_{1}, l_{2}} \delta_{x^{0}+\delta \tau, y^{0}}+t \delta \tau e^{\delta \tau \mu}\left[e^{i \delta \tau \varphi_{A}(x)} \delta_{l_{1}, A}, \delta_{l_{2}, B} \sum_{i=1}^{3} \delta_{x^{0}+\delta \tau, y^{0}} \delta_{\vec{x}+\vec{\rho}_{i}, \vec{y}}+e^{i \delta \tau \varphi_{B}(x)} \delta_{l_{1}, B} \delta_{l_{2}, A}\right.\right. \\
&\left.\left.\times \sum_{i=1}^{3} \delta_{x^{0}+\delta \tau, y^{0}} \delta_{\vec{x}-\vec{\rho}_{i}, \vec{y}}\right]+m c^{2} \delta \tau e^{\delta \tau \mu}\left[e^{i \delta \tau \varphi_{A}(x)} \delta_{l_{1}, A} \delta_{l_{2}, A} \delta_{\vec{x}, \vec{y}} \delta_{x^{0}+\delta \tau, y^{0}}-e^{i \delta \tau \varphi_{B}(x)} \delta_{l_{1}, B} \delta_{l_{2}, B} \delta_{\vec{x}, \vec{y}} \delta_{x^{0}+\delta \tau, y^{0}}\right]\right), \\
& \mathcal{S}_{e m}(\varphi)=\frac{\delta \tau}{2} \sum_{x, y} \varphi(x) V_{x y}^{-1} \varphi(y) .
\end{aligned}
$$

To get the expression for the fermion operator $\bar{M}$, one should take the formula for the $M$ and carry out the substitution $\varphi \rightarrow-\varphi, \mu \rightarrow-\mu$. Note that in formula (23) the summation is taken over all the coordinates $\left(\xi_{1}, \xi_{2}\right)$ and over the sublattice indices, so the interaction potential $V_{x y}$ is assumed to be a matrix,

$$
V_{x y}=\left(\begin{array}{ll}
V^{A A}(x, y) & V^{A B}(x, y) \\
V^{B A}(x, y) & V^{B B}(x, y)
\end{array}\right) .
$$

In the limit of $\delta \tau \rightarrow 0$, expression (21) corresponds to the partition function of the graphene sheet $L_{x} \times L_{y}$ at temperature $T$. In some applications, for instance, in the Monte Carlo simulation of graphene [24,25], one uses a small but finite step $\delta \tau$. Some of the formulas below are written for the finite $\delta \tau$ to have the possibility to estimate the discretization uncertainty.

\section{E. Propagators}

Using the expression for the partition function (21), one can write down the free propagators for the corresponding fields (see Fig. 2).

(1) The electron propagator,

$$
\begin{aligned}
\langle\eta(y) \bar{\eta}(x)\rangle & =M_{0}^{-1}(x, y) \\
& =\frac{1}{L_{t} L_{x} L_{y}} \sum_{k^{0}, \vec{k}} \frac{e^{i k(x-y)}}{\left(1-e^{i k^{0} \delta \tau} e^{\delta \tau \mu}\right)^{2}-\delta \tau^{2} E^{2}(\vec{k}) e^{2 i k^{0} \delta \tau} e^{2 \delta \tau \mu}}\left(\begin{array}{cc}
1-\left(1+m c^{2} \delta \tau\right) e^{i k^{0} \delta \tau} e^{2 \delta \tau \mu} & \varphi(\vec{k}) \delta \tau e^{i k^{0} \delta \tau} \\
\varphi^{*}(\vec{k}) \delta \tau e^{i k^{0} \delta \tau} & 1-\left(\begin{array}{cc}
\left.1-m c^{2} \delta \tau\right) e^{i k^{0} \delta \tau} e^{\delta \tau \mu}
\end{array}\right),
\end{array}\right.
\end{aligned}
$$

where $\varphi(\vec{k})$ was introduced in $(17)$.

Note that the matrix $M_{0}^{-1}(x, y)$ is the $2 \times 2$ matrix in the "sublattice space,"

$$
M_{0}^{-1}(x, y)=\left(\begin{array}{ll}
M_{0}^{A A}(x, y) & M_{0}^{A B}(x, y) \\
M_{0}^{B A}(x, y) & M_{0}^{B B}(x, y)
\end{array}\right)
$$

and its elements correspond to the propagation between one sublattice ( $A A$ and $B B$ ) and different sublattices $(A B$ and $B A)$.

(2) The hole propagator $\langle\eta(y) \bar{\eta}(x)\rangle$ can be obtained from the electron propagator with the substitution $\mu \rightarrow-\mu$. 
(3) The propagator of the "photon" field $\varphi$,

$$
\left\langle\varphi\left(\vec{x}, x^{0}\right) \varphi\left(\vec{y}, y^{0}\right)\right\rangle=\frac{1}{\delta \tau} \delta_{x^{0}, y^{0}} \hat{V}(\vec{x}, \vec{y})=\frac{1}{\delta \tau L_{\tau} L_{x} L_{y}} \sum_{k^{0}, \vec{k}} e^{i k(x-y)}\left(\begin{array}{cc}
\tilde{V}^{A A}(\vec{k}) & \tilde{V}^{A B}(\vec{k}) \\
\tilde{V}^{B A}(\vec{k}) & \tilde{V}^{B B}(\vec{k})
\end{array}\right) .
$$

The potential is instantaneous and acts in one Euclidean time slice.

Using the expressions for the partition function, for the electron and hole propagators one can show that, in the limit $\delta \tau \rightarrow 0$, the charge of graphene sheet $\langle Q\rangle$ is

$$
\langle Q\rangle=-\sum_{x}\left[\operatorname{Tr}\left(M_{x x}^{-1}\right)-\operatorname{Tr}\left(\bar{M}_{x x}^{-1}\right)\right]=2 \sum_{\vec{k}}\left(\frac{1}{e^{\beta(E(\vec{k})-\mu)}+1}-\frac{1}{e^{\beta(E(\vec{k})+\mu)}+1}\right),
$$

as it should be.

\section{F. Vertices}

The partition function (21) takes into account the interactions between electrons/holes and "photons." The interaction vertices of "photons" and electrons can be derived with an arbitrary accuracy through the expansion of the expression for the fermion operator:

$$
\begin{aligned}
\delta \mathcal{M}_{l_{1}, l_{2}}(x, y)= & \left(e^{i \delta \tau \varphi_{l_{1}}(x)}-1\right) e^{\delta \tau \mu}\left(-\delta_{\vec{x}, \vec{y}} \delta_{l_{1}, l_{2}} \delta_{x^{0}+\delta \tau, y^{0}}+t \delta \tau\left[\delta_{l_{1}, A}, \delta_{l_{2}, B} \sum_{i=1}^{3} \delta_{x^{0}+\delta \tau, y^{0}} \delta_{\vec{x}+\vec{\rho}_{i}, \vec{y}}\right.\right. \\
& \left.\left.+\delta_{l_{1}, B} \delta_{l_{2}, A} \sum_{i=1}^{3} \delta_{x^{0}+\delta \tau, y^{0}} \delta_{\vec{x}-\vec{\rho}_{i}, \vec{y}}\right]+m c^{2} \delta \tau\left[\delta_{l_{1}, A} \delta_{l_{2}, A} \delta_{\vec{x}, \vec{y}} \delta_{x^{0}+\delta \tau, y^{0}}-\delta_{l_{1}, B} \delta_{l_{2}, B} \delta_{\vec{x}, \vec{y}} \delta_{x^{0}+\delta \tau, y^{0}}\right]\right),
\end{aligned}
$$

in powers of $\delta \tau$. The simplest interaction vertex is shown in Fig. 3. Using formula (27), one can show that, at the leadingorder approximation in the $\delta \tau$ expansion, this vertex can be written as follows,

$$
V^{(3)}=(-i \delta \tau) \sum_{l_{1}, l_{2}, x, y}\left(\delta_{\vec{x}, \vec{y}} \delta_{l_{1}, l_{2}} \delta_{x^{0}+\delta \tau, y^{0}}\right) \bar{\psi}_{l_{1}}(x) \varphi_{l_{1}}(x) \psi_{l_{2}}(y),
$$

where $l_{1}, l_{2}$ are the sublattice indices.

The next vertex, which is required for the subsequent analysis and describes the interaction between two electrons and two "photons," is shown in Fig. 4. At the leading-order approximation it can be written as

$$
V^{(4)}=\frac{\delta \tau^{2}}{2} \sum_{l_{1}, l_{2}, x, y}\left(\delta_{\vec{x}, \vec{y}} \delta_{l_{1}, l_{2}} \delta_{x^{0}+\delta \tau, y^{0}}\right) \bar{\psi}_{l_{1}}(x) \varphi_{l_{1}}^{2}(x) \psi_{l_{2}}(y) .
$$

There are also additional vertices, coupling electrons to $3,4 \ldots, n, \ldots$ "photons" (because the $\varphi$ field stands in the exponent). However, they are suppressed by the additional

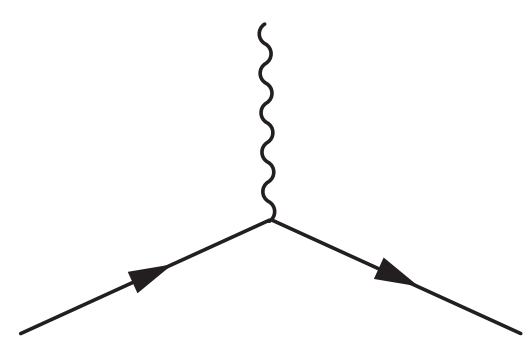

FIG. 3. The interaction vertex of electrons with one "photon" field. factors $\delta \tau$ and give no contribution to the final answer in the limit $\delta \tau \rightarrow 0$. Note also that in this section we presented only vertices with electrons. The vertices with holes can be found similarly.

A tight-binding model for interacting electrons on a hexagonal lattice with retarded Coulomb interactions, zero temperature, and zero chemical potential was introduced and studied in Refs. [31,32]. In this section we generalized some results of these papers to the case of an arbitrary interaction potential, nonzero temperature, and nonzero chemical potential.

\section{ONE-LOOP CORRECTIONS TO THE ELECTRON PROPAGATOR}

First, we are going to consider one-loop corrections at zero chemical potential and nonzero temperature. One-loop corrections to the electron propagator can be expressed in terms of the self-energy function $\Sigma(p)$,

$$
M^{-1}(p)=\frac{1}{M_{0}(p)-\Sigma(p)} .
$$

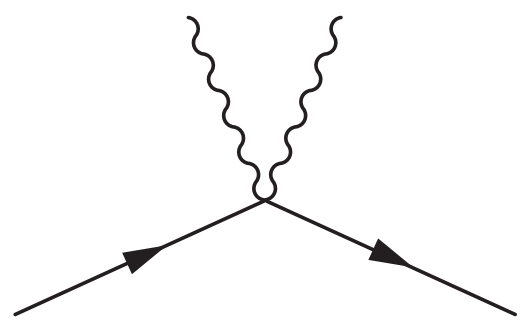

FIG. 4. The interaction vertex of electrons with two "photon" fields. 


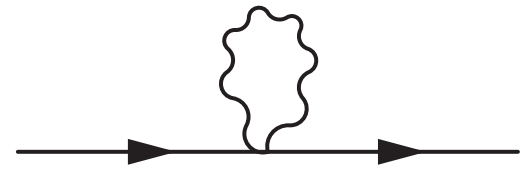

FIG. 5. Diagram that contributes to the self-energy $\Sigma_{1}(p)$.

At the leading-order approximation there are two diagrams shown in Figs. 5 and 6 that contribute to $\Sigma(p)$ :

$$
\begin{aligned}
\Sigma_{1}(p)= & +\frac{1}{2} \delta \tau\left(\begin{array}{cc}
V_{00} & 0 \\
0 & V_{00}
\end{array}\right) e^{i p^{0} \delta \tau}, \\
\Sigma_{2}(p)= & -\Sigma_{1}(p)+\frac{e^{i p^{0} \delta \tau} \delta \tau}{2 L_{x} L_{y}} \sum_{\vec{k}} \tanh \left(\frac{E(\vec{k})}{2 T}\right) \\
& \times\left(\begin{array}{cc}
-\frac{m c^{2}}{E(\vec{k})} \tilde{V}^{A A}(\vec{p}-\vec{k}) & \frac{\varphi(\vec{k})}{E(\vec{k})} \tilde{V}^{A B}(\vec{p}-\vec{k}) \\
\frac{\varphi^{*}(\vec{k})}{E(\vec{k})} \tilde{V}^{B A}(\vec{p}-\vec{k}) & \frac{m c^{2}}{E(\vec{k})} \tilde{V}^{B B}(\vec{p}-\vec{k})
\end{array}\right) .
\end{aligned}
$$

The inverse of the free electron propagator $M_{0}(p)$ can be written in the following form [see formula (25)]:

$$
\begin{aligned}
M_{0}(p)= & \left(\begin{array}{cc}
1-e^{i p^{0} \delta \tau} & 0 \\
0 & 1-e^{i p^{0} \delta \tau}
\end{array}\right) \\
& +e^{i p^{0} \delta \tau} \delta \tau\left(\begin{array}{cc}
m c^{2} & \varphi(\vec{p}) \\
\varphi^{*}(\vec{p}) & -m c^{2}
\end{array}\right),
\end{aligned}
$$

The final expression for $\Sigma(p)$ is

$$
\begin{aligned}
\Sigma(p)= & \Sigma_{1}(p)+\Sigma_{2}(p) \\
= & \frac{e^{i p^{0} \delta \tau} \delta \tau}{2 L_{x} L_{y}} \sum_{\vec{k}} \tanh \left(\frac{E(\vec{k})}{2 T}\right) \\
& \times\left(\begin{array}{cc}
-\frac{m c^{2}}{E(\vec{k})} \tilde{V}^{A A}(\vec{p}-\vec{k}) & \frac{\varphi(\vec{k})}{E(\vec{k})} \tilde{V}^{A B}(\vec{p}-\vec{k}) \\
\frac{\varphi^{*}(\vec{k})}{E(\vec{k})} \tilde{V}^{B A}(\vec{p}-\vec{k}) & \frac{m c^{2}}{E(\vec{k})} \tilde{V}^{B B}(\vec{p}-\vec{k})
\end{array}\right) .
\end{aligned}
$$

From formula (32) one can see that one-loop corrections are reduced to the renormalization of the mass $m$ and the function $\varphi(\vec{p})$. The renormalized mass now depends on the point in the Brillouin zone and has the form

$$
\begin{aligned}
m^{R}(\vec{p})= & m+\frac{m}{2 L_{x} L_{y}} \sum_{\vec{k}} \tanh \left(\frac{E(\vec{k})}{2 T}\right) \\
& \times \frac{1}{E(\vec{k})} \tilde{V}^{A A}(\vec{p}-\vec{k}) .
\end{aligned}
$$

The expression for the renormalized function $\varphi^{R}(\vec{p})$ is

$$
\begin{aligned}
\varphi^{R}(\vec{p})= & \varphi(\vec{p})+\frac{1}{2 L_{x} L_{y}} \sum_{\vec{k}} \tanh \left(\frac{E(\vec{k})}{2 T}\right) \\
& \times \frac{\varphi(\vec{k})}{E(\vec{k})} \tilde{V}^{A B}(\vec{p}-\vec{k}) .
\end{aligned}
$$

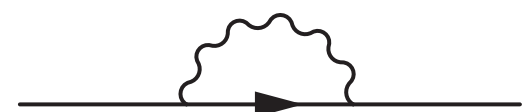

FIG. 6. Fock diagram that contributes to the self-energy $\Sigma_{2}(p)$
Thus one-loop corrections conserve the form of the propagator (25) and substitute the free mass $m$ and the function $\varphi(\vec{p})$ with the renormalized expressions (33) and (34). From this one can conclude that the energy spectrum of the quasiparticles at the one-loop approximation is $E^{2}=\left(m^{R} c^{2}\right)^{2}+\left(\varphi^{R}\right)^{2}$. In order to estimate the size of one-loop corrections, in Fig. 7 we plot the energy spectrum profile of electrons with Coulomb, screened Coulomb [23] interactions, and without interactions. The calculation was carried out at $T=0.1 \mathrm{eV}$ at an infinitely large lattice.

Formulas (33) and (34) can be used to reproduce wellknown results of the effective theory of graphene and generalize them to the case of nonzero temperature. To this end, we consider large lattice $L_{x}, L_{y} \rightarrow \infty$ with the Coulomb interactions between electrons near the Fermi point. Then formulas (33) and (34) can be written as

$$
\begin{aligned}
m^{R}\left(\vec{p}=\vec{p}_{F}\right)= & m\left(1+\frac{1}{2} \frac{\alpha}{\left(v_{F} / c\right)}\left[\log \left(\frac{\Lambda}{2 T}\right)+\gamma\right.\right. \\
& \left.\left.-\log \pi / 4+O\left(\Lambda^{-1}\right)\right]\right), \\
v_{F}^{R}= & v_{F}\left(1+\frac{1}{4} \frac{\alpha}{\left(v_{F} / c\right)}\left[\log \left(\frac{\Lambda}{2 T}\right)+\gamma\right.\right. \\
& \left.\left.-\log \pi / 4+O\left(\Lambda^{-1}\right)\right]\right),
\end{aligned}
$$

where $\Lambda$ is the ultraviolet cutoff, and $\gamma \approx 0.577 \ldots$ is the Euler's constant. These formulas are in agreement with the predictions of the effective theory of graphene at the leading logarithmic accuracy $[14,35]$.

During the derivation of formulas (35) we carried out the integration over the two-dimensional sphere of radius $\Lambda$. It was also assumed that $m c^{2} \ll T, \Lambda$ is much larger than all other energy scales involved. Note that the theory is regularized by the temperature in the infrared region. Note also that the ratio of the ultraviolet and the infrared cutoffs $\Lambda / T$ under the logarithm is multiplied by the $v_{F} / c$ that considerably reduces the total renormalization of the Fermi velocity.

In order to study the finite density effects, we consider one-loop corrections at zero temperature and nonzero chemical potential. In addition to the diagrams shown in Figs. 5 and 6, there is a contribution of the interaction of the electron propagator with vacuum represented by the diagram shown in Fig. 8. As before, one-loop corrections at nonzero $\mu$ lead to the renormalization of the parameters of free propagator, preserving its structure. In particular, the mass and the function $\varphi$ at one-loop approximations can be written as follows:

$$
\begin{aligned}
& m^{R}(\vec{p})=m+\frac{m}{2 L_{x} L_{y}} \sum_{\vec{k}} \frac{1}{E(\vec{k})} \tilde{V}^{A A}(\vec{p}-\vec{k}) \theta(E(\vec{k})-\mu), \\
& \varphi^{R}(\vec{p})=\varphi(\vec{p})+\frac{1}{2 L_{x} L_{y}} \sum_{\vec{k}} \frac{\varphi(\vec{k})}{E(\vec{k})} \tilde{V}^{A B}(\vec{p}-\vec{k}) \theta(E(\vec{k})-\mu) .
\end{aligned}
$$

In the limit of large lattice $L_{x}, L_{y} \rightarrow \infty$, the Coulomb interaction and the linear electron spectrum near the Fermi 


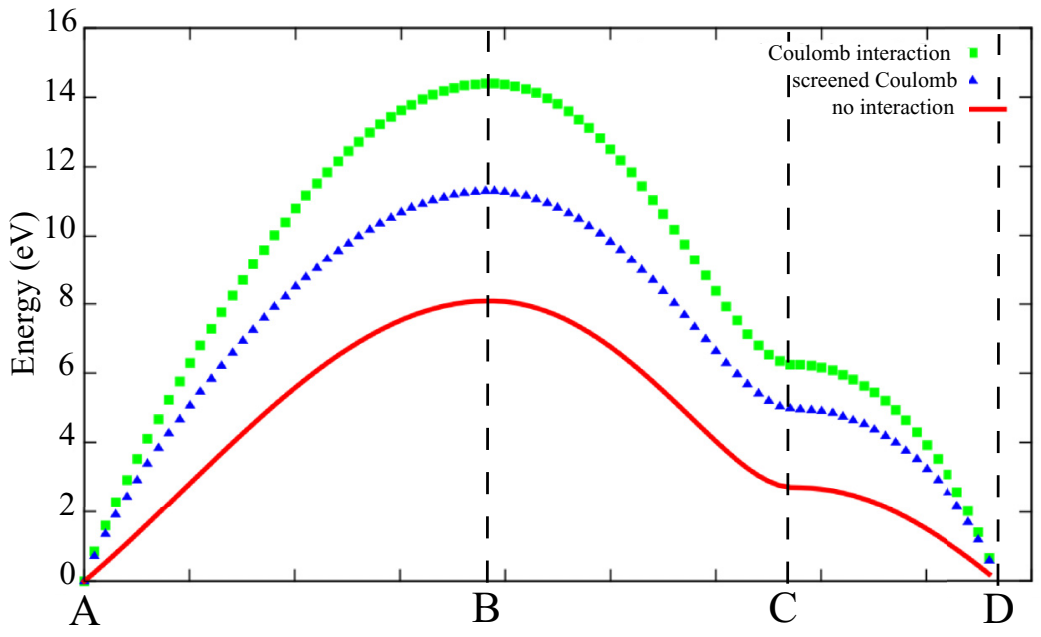

(a)

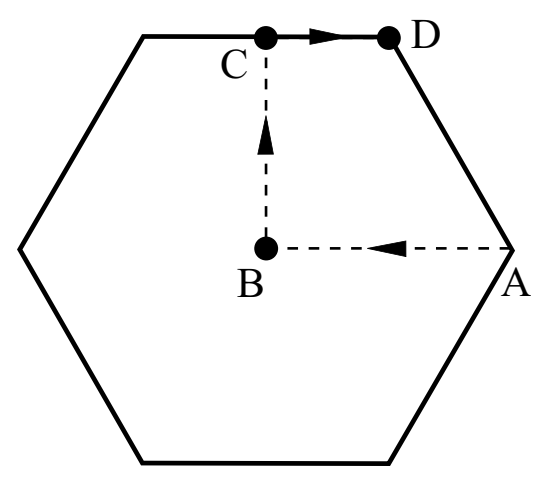

(b)

FIG. 7. (Color online) Energy spectrum profile of electrons with Coulomb, screened Coulomb [23] interactions, and without interactions. The calculation was carried out at $T=0.1 \mathrm{eV}$ at an infinitely large lattice. (a) Free and renormalized energy $E(\vec{k})$ profile. (b) The spectrum profile is drawn on the polygon $A B C D$.

point, formulas (36) can be written as

$$
\begin{aligned}
m^{R}\left(\vec{p}=\vec{p}_{F}\right) & =m\left(1+\frac{1}{2} \frac{\alpha}{\left(v_{F} / c\right)} \log \left[\frac{\Lambda}{\mu}\right]\right), \\
v_{F}^{R} & =v_{F}\left(1+\frac{1}{4} \frac{\alpha}{\left(v_{F} / c\right)} \log \left[\frac{\Lambda}{\mu}\right]\right) .
\end{aligned}
$$

Similarly to the case of nonzero temperature, chemical potential $\mu$ plays the role of the infrared cutoff. It should be noted now that there are four scales that can play the role of the infrared cutoff: the fermion mass $m$, the chemical potential, the temperature, and the inverse lattice size. We believe that in the infrared limit the theory is regularized by the largest of these scales. Note that formulas (35) and (36) take these effects into account exactly.

Note also that formulas (35) can be written in the form (37) if instead of $\Lambda$ one uses the ultraviolet cutoff $\Lambda^{\prime}=$ $2 e^{\gamma} / \pi \Lambda \sim 1.13 \Lambda$ and substitute the chemical potential by the temperature.

For this reason, at temperatures equal to the chemical potential, the renormalization due to temperature effects is a little bit larger than the renormalization due to a nonzero chemical potential. In this consideration we did not take into account the Debye screening of the interaction potential at

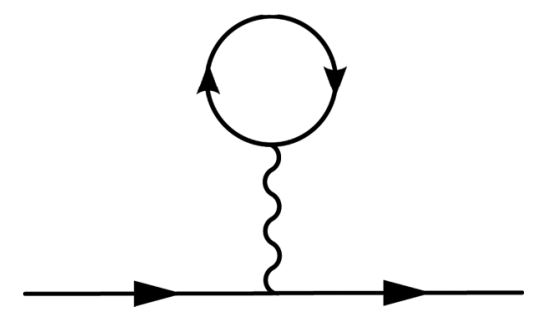

FIG. 8. Hartree diagram that contributes to the one-loop electron propagator at $\mu \neq 0$. large distances and the screening of the Coulomb potential at small distances, which will be done in the last section.

In Refs. [31,32] the tight-binding model was used to study the higher-order renormalization of the Fermi velocity and the emergence of critical exponents, using lattice Ward identities. In addition, the authors showed that the symmetry structure of the dressed fermion propagator is the same as that for the free propagator. One can also mention Ref. [27], where tight-binding methods were used to study the one-loop renormalization of the quasiparticle dispersion relation for Weyl semimetals. The results obtained in these papers are very similar to the results of this section. However, the studies carried out in Refs. [27,31,32] were done for a Coulomb interaction potential at zero temperature and zero chemical potential. So, the results of this section can be considered as a one-loop study of renormalization properties of the fermion propagator generalized to nonzero temperature, nonzero electron density, and arbitrary interaction potential. In addition, in this section we numerically calculated the renormalized spectrum of the fermion excitations for a realistic interaction potential, which was not done in Ref. [27].

\section{ONE-LOOP CORRECTIONS TO "PHOTON" PROPAGATOR AND DIELECTRIC PERMITTIVITY OF GRAPHENE}

In this section we calculate a one-loop correction to the instantaneous "photon" propagator $\left\langle\varphi\left(\vec{x}, x^{0}\right) \varphi\left(\vec{y}, y^{0}\right)\right\rangle$. Oneloop corrections to the propagator in the momentum space can be expressed in terms of the polarization operator $\hat{\mathcal{P}}=$ $\left(\begin{array}{ll}\mathcal{P}^{A A} & \mathcal{P}^{A B} \\ \mathcal{P}^{B A} & \mathcal{P}^{B B}\end{array}\right)$ as

$$
\begin{aligned}
\tilde{V}^{R}(\vec{k}) & =\frac{1}{\hat{\epsilon}(\vec{k})} \times \tilde{V}(\vec{k}), \\
\hat{\epsilon}(\vec{k}) & =1-\tilde{V}(\vec{k}) \times \hat{\mathcal{P}}(\vec{k}),
\end{aligned}
$$


where $\tilde{V}^{R}(\vec{k}), \tilde{V}(\vec{k})$ are renormalized and three-level Fourier transforms of the potential, which are the $2 \times 2$ matrices in the sublattice space. In the limit of $\delta \tau \rightarrow 0$ and one-loop approximation the only diagram shown in Fig. 9 contributes.

The expression for the polarization operator at one-loop approximation can be written in the following form:

$$
\begin{aligned}
\mathcal{P}^{A A}(\vec{k}) & =\mathcal{P}^{B B}(\vec{k})=\frac{1}{L_{x} L_{y}} \sum_{\vec{p}} \frac{1}{E^{2}(\vec{p}-\vec{k})-E^{2}(\vec{p})}\left(\frac{m^{2} c^{4}+E^{2}(\vec{p})}{E(\vec{p})} \tanh \frac{E(\vec{p})}{2 T}-\frac{m^{2} c^{4}+E^{2}(\vec{p}-\vec{k})}{E(\vec{p}-\vec{k})} \tanh \frac{E(\vec{p}-\vec{k})}{2 T}\right), \\
\mathcal{P}(\vec{k}) & =\mathcal{P}^{* B A}(\vec{k})=\frac{1}{L_{x} L_{y}} \sum_{\vec{p}} \frac{\varphi(\vec{p}) \varphi^{*}(\vec{p}-\vec{k})}{E^{2}(\vec{p}-\vec{k})-E^{2}(\vec{p})}\left(\frac{1}{E(\vec{p})} \tanh \frac{E(\vec{p})}{2 T}-\frac{1}{E(\vec{p}-\vec{k})} \tanh \frac{E(\vec{p}-\vec{k})}{2 T}\right) .
\end{aligned}
$$

Using formulas (38) and (39), one can show that at large distances and at small temperature, the expression for the interaction potentials for all the sublattice indices take the form

$$
\tilde{V}^{R}(\vec{k})=\frac{2 \pi \alpha c}{\epsilon_{0}} \frac{1}{\left(|\vec{k}|+m_{D} c\right)},
$$

where

$$
\begin{aligned}
\epsilon_{0} & =1+\frac{\pi}{2} \frac{\alpha}{\left(v_{F} / c\right)}, \\
m_{D} & =\frac{8 \alpha \log 2}{\epsilon_{0} v_{F}^{2}} T .
\end{aligned}
$$

Now, one may see that at zero temperature the interaction potential is the Coulomb potential screened by the dielectric permittivity $\epsilon_{0}$, which is in agreement with the RPA result [13]. At nonzero temperature there is a two-dimensional Debye screening of the Coulomb potential with the Debye mass $m_{D}$, which agrees with the results of Ref. [36].

Similarly, one can study the question of how the nonzero density acts on the dielectric permittivity of graphene. To get the expression for the polarization operator in this case one can use formulas (39) and carry out the following substitution, $\tanh (E / 2 T) \rightarrow \theta(E-\mu)$. It is not difficult to find out that at large distances the expression for the interaction potential has the form (40), with $\epsilon$ given by Eq. (41) and the Debye mass

$$
m_{D}=\frac{4 \alpha}{\epsilon_{0} v_{F}^{2}}|\mu| .
$$

The last expression agrees with the RPA result [13].

At the end of this section we plot the dielectric permittivity of graphene $\epsilon$ obtained from formulas (38) and (39) as a

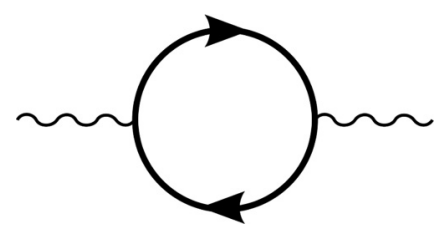

FIG. 9. Empty-loop diagram that contributes to the renormalization of the interaction potential. function of distance $r$ in units of the lattice spacing $a$ (Fig. 10). The calculation was carried out for suspended graphene with the interaction potential from Ref. [24] and for different external conditions: $T=0, \mu=0, T=0, \mu=0.026 \mathrm{eV}$ $\left(n \sim 6.5 \times 10^{10} \mathrm{~cm}^{-2}\right.$ ), and $T=0.026 \mathrm{eV}, \mu=0$. In order to compare our results with RPA, we plot the dielectric permittivity given by formula (41). For small distances we compared our results with the results of the Monte Carlo simulation [37]. It is seen that the Monte Carlo results are in a good agreement with one-loop results.

From Fig. 10 it is seen that at zero temperature the $\epsilon$ starting from the value $\sim 2$ at $r=0$ approaches the value $\epsilon_{0}$ (41) already for $r / a>5$. At nonzero temperature and chemical potential, the larger the $r$, the larger is the $\epsilon$. This behavior can be attributed to the Debye screening effect.

Renormalization properties of the photon propagator were studied in Ref. [32], where it was shown that the Coulomb interaction conserves the structure of the propagator. Our study is very different for that done in Ref. [32]. The point is that in our study either the chemical potential or temperature is different from zero. This leads to the appearance of the photon mass (Debye mass) and a dramatic change of the structure of the "photon" propagator.

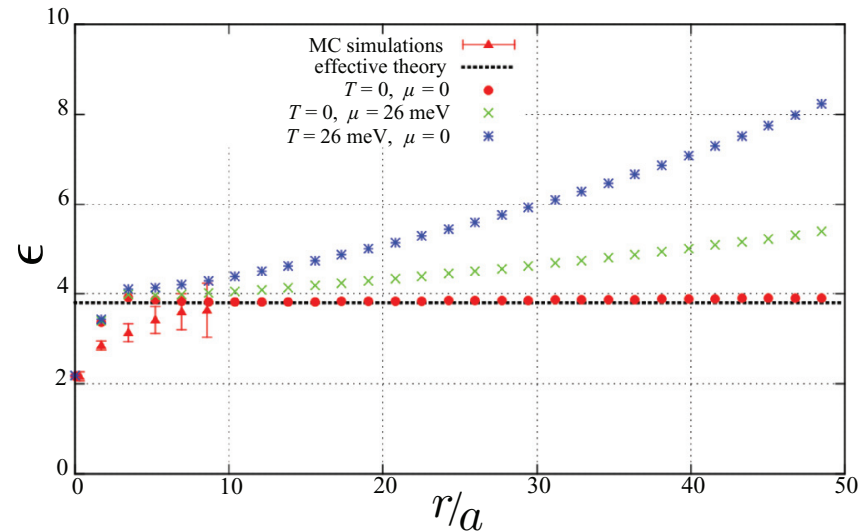

FIG. 10. (Color online) The dielectric permittivity of suspended graphene $\epsilon$ as a function of distance $r$ in units of distance between the neighboring carbon atoms $a$ for different external conditions. The dotted line corresponds to the RPA expression (41). The points shown as red triangles are the results of Monte Carlo simulations. 


\section{DISCUSSION AND CONCLUSION}

This paper is devoted to the perturbation theory which can be used for studying the properties of graphene at finite temperature and nonzero chemical potential. This perturbation theory is based on the tight-binding Hamiltonian on a hexagonal lattice and arbitrary interaction potential between electrons, which is considered as a perturbation. We built the partition function for this theory, and derived Feynman rules and expressions for free propagators.

As an example of the application, we calculated one-loop corrections to the electron propagator. It was shown that one-loop corrections lead to the renormalization of the bare mass and the function $\varphi(\vec{k})$ conserving the structure of the propagator. Using this result, we calculated the one-loop energy spectrum of electrons, renormalized fermion mass, and Fermi velocity.

In order to estimate the value of the renormalization, in Fig. 11 we plotted the renormalization factor for the Fermi velocity as a function of the temperature for graphene on hexagonal boron nitride (hBN) for the Coulomb and screened at small distance Coulomb interactions [23]. During the calculation we took into account the screening of the potentials by the dielectric permittivity (38). The results can be well described by the formula

$$
v_{F}^{R}(T) / v_{F}^{0}=\left(1+A \log \left[\frac{\Lambda}{\mu}\right]\right),
$$

where for the Coulomb interaction $A=0.096, \Lambda=3.2 \mathrm{eV}$, and for the screened Coulomb interaction $A=0.093, \Lambda=$ $2.4 \mathrm{eV}$. The effective ultraviolet cutoff $\Lambda$ is sensitive to the values of the potential at small distances, contrary to the coefficient $A$. Note also that that the coefficient $A$ is well described by the expression $A \approx \alpha c /\left(4 v_{F} \epsilon^{\prime}\right)=0.094$, $\epsilon^{\prime}=\left(\epsilon_{\mathrm{BN}}+1\right) / 2+\epsilon_{0}-1=6.7$.

In addition, we considered one-loop corrections to the electron propagator at zero temperature and nonzero chemical potential. It was shown that, similarly to the nonzero temperature case, one-loop corrections conserve the structure of the propagator leading to the renormalization of already existing parameters. In particular, we obtained the formulas for the

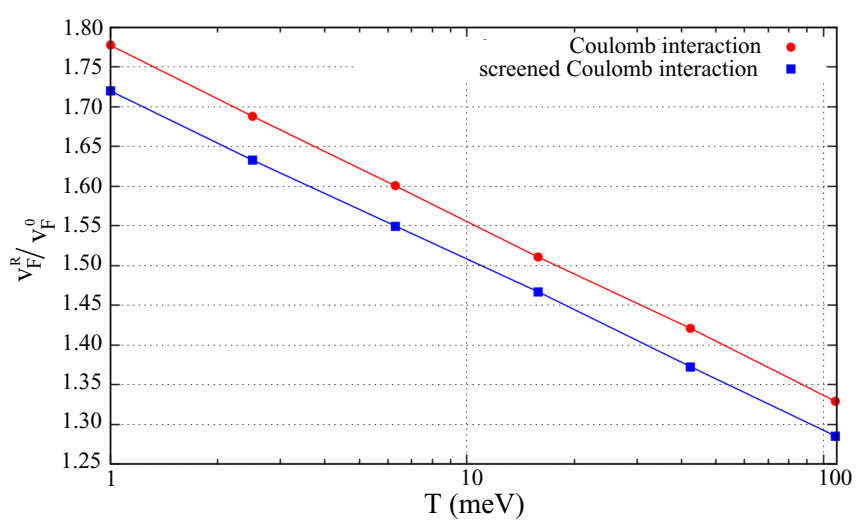

FIG. 11. (Color online) The renormalization factor for the Fermi velocity as a function of the temperature for graphene on $\mathrm{hBN}$ for Coulomb and screened at small distance Coulomb interactions [23]. renormalization of the Fermi velocity and the fermion mass in this case.

In Ref. [16] the renormalization of the Fermi velocity was studied through the measurement of the quantum capacitance at nonzero chemical potential. In this study the graphene layer was placed inside $\mathrm{hBN}$, which reduced the strength of the interaction between electrons. This allows us to expect that perturbation theory works well in this case, and we can compare our results with the result of Ref. [16]. The results of the measurements of the Fermi velocity can be well fitted by the formula

$$
\begin{aligned}
v_{F}(\mu) & =v_{F}\left(\mu_{0}\right)\left(1+\frac{1}{4} \frac{\alpha}{\epsilon\left(v_{F} / c\right)} \log \left[\frac{\mu_{0}}{\mu}\right]\right) \\
& =v_{F}\left(\mu_{0}\right)\left(1+A \log \left[\frac{\mu_{0}}{\mu}\right]\right),
\end{aligned}
$$

where $\quad v_{F}\left(\mu_{0}\right)=0.85 \times 10^{6} \mathrm{~m} / \mathrm{s}, \quad \mu_{0}=3.2 \mathrm{eV} \quad\left(n_{0}=\right.$ $\left.10^{15} \mathrm{~cm}^{-2}\right), \epsilon \simeq 8$, and $A=0.081$. Note that the original formula for the Fermi velocity from Ref. [16] depends on the density $n$. In (44) we turn the dependence on $n$ to the dependence on the chemical potential.

In the calculation we used formulas (36) with the interaction potential screened by the dielectric permittivity (38). The interaction potential at small distances was taken from Ref. [23] and divided by $\epsilon_{\mathrm{BN}} \simeq 4$.5. At large distances we took the Coulomb potential screened by $\epsilon_{\mathrm{BN}}$. The results of the calculations can be well fitted by formula (44) with the following parameters: $\mu_{0}=2.9 \mathrm{eV}, A=0.072$. These values are in reasonable agreement with Ref. [16]. In addition, we carried out the calculation of the parameters $\mu_{0}, A$ for the Coulomb potential screened by $\epsilon_{\mathrm{BN}}$. Our result is $\mu_{0}=5.2 \mathrm{eV}$, $A=0.077$. Again, we see that the value of the constant $\mu_{0}$ is sensitive to the values of the potential at small distances.

It is also interesting to compare the renormalization of the Fermi velocity due to nonzero $\mu$ and nonzero temperature. To this end we calculated the nonzero temperature renormalization of the graphene layer placed inside the $\mathrm{hBN}$ for the Coulomb and screened at small distance Coulomb interactions. The results can be described by formula (43) with the following parameters: for the Coulomb interaction $A=0.075, \Lambda=$ $4.5 \mathrm{eV}$, and for the screened Coulomb interaction, $A=0.073$, $\Lambda=3.05 \mathrm{eV}$.

The other example of application of the perturbation theory is the calculation of one-loop corrections to the interaction potential done in the previous section. We derived the oneloop expression for the dielectric permittivity at nonzero temperature, nonzero chemical potential, and the arbitrary interaction potential.

It is well known that the electrons in graphene form a strongly interacting system. So it is reasonable to consider the question of how our results are affected by the higherorder corrections. The authors of Ref. [22] considered the next-to-leading order (NLO) corrections to the Fermi velocity within the effective theory of graphene. The main result of this paper is the statement that if one expands the Fermi velocity renormalization in the one-loop RPA potential instead of the usual Coulomb potential, the NLO corrections to the leading-order (LO) result (37) turn out to be small. This allows us to expect a good accuracy of formulas (33), (34), and (36) 
with the one-loop potential given by formulas (38) and (39), even for suspended graphene.

The authors of Ref. [21] considered the NLO corrections to the polarization operator within the effective theory of graphene. The NLO value of the dielectric permittivity for suspended graphene is approximately $30 \%$ smaller than the LO result, which is not very large. Moreover, the Monte Carlo results [37] tell us that the higher-order corrections to the LO result can be even smaller than $30 \%$. For this reason one can expect that the accuracy of formulas (38) and (39) is rather good.

Finally, we would like to mention Ref. [27], where the authors noticed the fact that the tight-binding approach provides a quantitative prediction for the ultraviolet cutoff $\Lambda$ and estimated its value for the Weyl semimetals as $\Lambda \sim 0.53 \pi / a$.
If we use this formula to estimate $\Lambda$ in graphene, we will get $\Lambda=6.7 \mathrm{eV}$, which is by a factor of $\sim 2$ larger than our estimates. The difference in the values of $\Lambda$ can be attributed to the fact that the effective ultraviolet cutoff is very sensitive to the screening effects and a realistic interaction potential that was not accounted for in Ref. [27].

\section{ACKNOWLEDGMENTS}

We would like to thank M. Ulybyshev and A. Nikolaev for interesting discussions. M.I.K. acknowledges financial support from NWO via Spinoza Prize. The work of V.V.B. and N.Y.A. was supported by the Far Eastern Federal University, by RFBR Grants No. 14-02-01261-a, No. 15-02-07596, and No. 15-3221117, and the Dynasty Foundation.
[1] K. S. Novoselov, A. K. Geim, S. V. Morozov, D. Jiang, Y. Zhang, S. V. Dubonos, I. V. Grigorieva, and A. A. Firsov, Science 306, 666 (2004).

[2] A. K. Geim and K. S. Novoselov, Nat. Mater. 6, 183 (2007).

[3] P. R. Wallace, Phys. Rev. 71, 622 (1947).

[4] J. W. McClure, Phys. Rev. 104, 666 (1956).

[5] G. W. Semenoff, Phys. Rev. Lett. 53, 2449 (1984).

[6] K. S. Novoselov, A. K. Geim, S. V. Morozov, D. Jiang, M. I. Katsnelson, I. V. Grigorieva, S. V. Dubonos, and A. A. Firsov, Nature (London) 438, 197 (2005).

[7] Y.Zhang, Y.-W. Tan, H. L. Stormer, and P. Kim, Nature (London) 438, 201 (2005).

[8] M. I. Katsnelson and K. S. Novoselov, Solid State Commun. 143, 3 (2007).

[9] C. W. J. Beenakker, Rev. Mod. Phys. 80, 1337 (2008).

[10] A. H. Castro Neto, F. Guinea, N. M. R. Peres, K. S. Novoselov, and A. K. Geim, Rev. Mod. Phys. 81, 109 (2009).

[11] M. A. H. Vozmediano, M. I. Katsnelson, and F. Guinea, Phys. Rep. 496, 109 (2010).

[12] V. N. Kotov, B. Uchoa, V. M. Pereira, F. Guinea, and A. H. Castro Neto, Rev. Mod. Phys. 84, 1067 (2012).

[13] M. I. Katsnelson, Graphene: Carbon in Two Dimensions (Cambridge University Press, Cambridge, UK, 2012).

[14] J. Gonzalez, F. Guinea, and M. A. H. Vozmediano, Nucl. Phys. B 424, 595 (1994).

[15] D. C. Elias, R. V. Gorbachev, A. S. Mayorov, S. V. Morozov, A. A. Zhukov, P. Blake, L. A. Ponomarenko, I. V. Grigorieva, K. S. Novoselov, F. Guinea, and A. K. Geim, Nat. Phys. 7, 701 (2011).

[16] G. L. Yu, R. Jalil, B. Belle, A. S. Mayorov, P. Blake, F. Schedin, S. V. Morozov, L. A. Ponomarenko, F. Chiappini, S. Wiedmann, U. Zeitler, M. I. Katsnelson, A. K. Geim, K. S. Novoselov, and D. C. Elias, Proc. Natl. Acad. Sci. USA 110, 3282 (2013).

[17] E. G. Mishchenko, Phys. Rev. Lett. 98, 216801 (2007).

[18] I. F. Herbut, V. Juričić, and O. Vafek, Phys. Rev. Lett. 100, 046403 (2008).

[19] L. Fritz, J. Schmalian, M. Muller, and S. Sachdev, Phys. Rev. B 78, 085416 (2008).
[20] F. de Juan, A. G. Grushin, and M. A. H. Vozmediano, Phys. Rev. B 82, 125409 (2010).

[21] I. Sodemann and M. M. Fogler, Phys. Rev. B 86, 115408 (2012).

[22] J. Hofmann, E. Barnes, and S. Das Sarma, Phys. Rev. Lett. 113, 105502 (2014).

[23] T. O. Wehling, E. Sasioglu, C. Friedrich, A. I. Lichtenstein, M. I. Katsnelson, and S. Blugel, Phys. Rev. Lett. 106, 236805 (2011).

[24] M. V. Ulybyshev, P. V. Buividovich, M. I. Katsnelson, and M. I. Polikarpov, Phys. Rev. Lett. 111, 056801 (2013).

[25] P. V. Buividovich and M. I. Polikarpov, Phys. Rev. B 86, 245117 (2012).

[26] D. Smith and L. von Smekal, Phys. Rev. B 89, 195429 (2014).

[27] B. Rosenstein, M. Lewkowicz, and T. Maniv, Phys. Rev. Lett. 110, 066602 (2013).

[28] J. E. Drut and T. A. Lähde, Phys. Rev. Lett. 102, 026802 (2009); Phys. Rev. B 79, 165425 (2009); 79, 241405 (2009).

[29] W. Armour, S. Hands, and C. Strouthos, Phys. Rev. B 81, 125105 (2010); 84, 075123 (2011).

[30] P. V. Buividovich, E. V. Luschevskaya, O. V. Pavlovsky, M. I. Polikarpov, and M. V. Ulybyshev, Phys. Rev. B 86, 045107 (2012).

[31] A. Giuliani, V. Mastropietro, and M. Porta, Phys. Rev. B 82, 121418(R) (2010).

[32] A. Giuliani, V. Mastropietro, and M. Porta, Ann. Phys. 327, 461 (2012).

[33] A. Giuliani, V. Mastropietro, and M. Porta, Phys. Rev. B 83, 195401 (2011).

[34] B. Rosenstein and M. Lewkowicz, Phys. Rev. B 88, 045108 (2013).

[35] V. N. Kotov, V. M. Pereira, and B. Uchoa, Phys. Rev. B 78, 075433 (2008).

[36] V. V. Braguta, S. N. Valgushev, A. A. Nikolaev, M. I. Polikarpov, and M. V. Ulybyshev, Phys. Rev. B 89, 195401 (2014).

[37] N. Yu. Astrakhantsev, V. V. Braguta, M. I. Katsnelson, A. A. Nikolaev, M. V. Ulybyshev, and O. E. Soloveva (unpublished). 